\title{
A Fuzzy Logic Based System for Mixed Reality Assistance of Remote Workforce
}

\author{
Anasol Peña-Rios, Hani Hagras, Michael Gardner \\ Intelligent Environments Research Group, \\ School of Computer Science and Electronic Engineering, \\ University of Essex, Wivenhoe Park, \\ Colchester, CO4 3SQ, UK.
}

\author{
Gilbert Owusu \\ Business Modelling and Operational Transformation \\ Practice, British Telecomm, Adastral Park, Martlesham \\ Heath, Ipswich, UK
}

\begin{abstract}
The recent years have witnessed an increase in the use of augmented and virtual reality systems, changing the way we interact with our environments. Such systems are commonly associated with advertising, entertainment, medicine, training and education. However, with the increasing acceptance and availability of mobile and wearable devices (e.g. head-mounted displays (HMD)), the use of these technologies is moving towards professional and industrial environments, where they would be able to support employees in their daily tasks, increasing customer satisfaction and reducing business costs. This paper presents an innovative Mixed Reality (MR) system to assist field workforce in remote locations. As part of the overall implementation, the MR system uses fuzzy logic mechanisms to improve accuracy in user tracking and object monitoring, allowing the correct representation of users and objects in the Graphical User Interfaces (GUIs), and improving the experience for users.
\end{abstract}

Keywords-fuzzy logic system; mixed reality; augmented reality;

\section{INTRODUCTION}

The increasing complexity of tasks and specialised equipment in different industries, such as aeronautic, automobile, manufacturing and telecommunications industries, have triggered the need of trained specialists and workforce to perform daily activities. Training specialized workers represents a considerable overhead in any business. As an example, it takes up to 2000 hours for aeronautic inspectors to complete their training [1]. This creates the need of human-centred assistance systems to support employees in their tasks, and help them to improve their skills. The use of Mixed Reality (MR) technologies, such as Augmented Reality (AR) and Virtual Reality (VR), can potentially enhance working environments by removing restrictions of time and location, and leading to a much faster knowledge transfer and a better understanding of different processes. Furthermore, industry can benefit from the use of mixed reality to lower operational costs and thus sustain their growth and innovation.

In particular, AR systems have become more used in professional and industrial environments due to the increasing growth of mobile devices. In addition, the use of ubiquitous computing as an infrastructure for mixed reality has opened the possibility of having it pervasively in our daily lives, allowing collaboration between users in different geographical locations. A great deal of handheld devices, such as smartphones and tablets, integrate cameras and sensors (e.g. accelerometers, gyroscopes, compasses, GPS, etc.), that can easily be turned into
AR systems. Head mounted displays (HMD) (e.g. AR glasses), although not that common, provide similar sensing capabilities with a higher level of immersion for the user. AR solutions have been used in different industries such as textile [2], aeronautic [1], medical care [3], manufactory [4] [5], military [6], and architectural construction [7] to support workers in their activities.

Another possible application for AR is in Customer Service, where customers instead of waiting next to the phone to receive instructions when setting up technology at home, or have to wait for a visit of an engineer to do configuration or installation of devices and services; they could use their mobile devices, and overlay instructions through the camera in real-time, with a technician guiding him/her through the process. Then, instead of hearing generic instructions, like "plug the black wire" or "check that the port is enabled", customers and technicians could share the same view; with technicians providing better guidance in helping users to solve problems, improving customer satisfaction, and saving resources as technicians and engineers do not need to travel to customers' location. The same scenario could be applied for technicians and engineers with different levels of training and expertise. In this case, a young trainee could benefit from the remote guidance and help of a more experienced engineer.

This paper presents an approach based on mixed-reality and fuzzy logic to assist field workforce when performing on-site activities. The proposed platform assists workers in the execution of their daily tasks by connecting them with experts situated in a control centre. When one on-site worker identifies a non-familiar issue, he/she can get assistance from an expert in the control centre. Using Inertial Measurement Unit (IMU) sensors located in a mobile phone, the mixed reality assistance system detects worker position, and sends this information to the control centre, along with current information of the device he/she is working on; sharing with the expert the situation that he/she is experiencing. In like manner, the device is monitored in real-time by the control centre to help the user to determine the possible cause of the problem.

The rest of the paper is structured as follows: section II presents a brief introduction to MR systems and the current challenges in this technology. Section III introduces an innovative model for a fuzzy logic based mixed-reality assistance system for remote field workforce, explaining the conceptual model and architecture behind as well as describing 
our current implementation. Section IV describes the experiments and results and the conclusions and future work are presented in Section V.

\section{A BRIEF OVERVIEW ON MIXED REALITY}

Mixed Reality (MR) can be defined as the spectrum that connects the physical world (absent from virtual representations of any kind) to the virtual world, allowing the co-existence of physical and computer-generated elements in real-time. Milgram and Kishino [8] proposed a continuum (shown in Fig.1) to represent the different degrees between reality and virtuality, defining anything amid the ends as Mixed Reality.

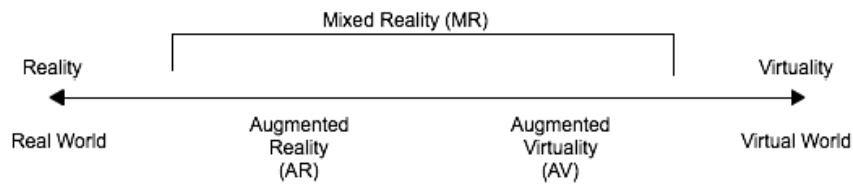

Fig. 1. Reality-Virtuality Continuum [8].

Augmented Reality (AR) describes an environment where the physical world is enhanced by adding computer-generated objects to make them appear as if they co-exist in the same dimension [9]. Its potential relies on the possibility of enhancing reality, making invisible things visible [9] and sometimes, due to its synthetic nature, modifying the physical laws governing reality by implementing diverse metaphors (visual, auditory and haptic) not available in the physical world [10]. Therefore, AR supplements reality rather than completely replacing it (as VR does). Hence, AR presents information that is not directly detected by users' senses, helping them to perform real-world tasks, and facilitating the understanding of complex scenarios [11]. To achieve this, AR uses interfaces with minimal rendering that require high accuracy in tracking and sensing [12]. Tracking is the activity of locating user's position and orientation in reference to an environment [13]. Currently, tracking is generally based on two approaches: the use of computer vision techniques or via inertial motion devices, such as accelerometers and gyroscopes, available in mobile and wearable devices. An accelerometer can be specified as a single-degree-of-freedom device that measures the linear acceleration of an object to which it is attached [14]. Gyroscopes are used to measure angular velocity, based on the principle of conservation of the angular momentum that states that an object rotated at high angular speed in the absence of external moments, conserves its angular momentum [14]. These devices are used in inertial navigation systems for aircraft, spacecraft, Unmanned Aerial Vehicle (UAV), among others, where the need for accuracy is vital. Inertial tracking is faster and more robust when rapid changes occur compared to vision-based tracking methods [12]. Inertial tracking systems need to handle uncertainties associated with dynamic environments such as noise, and wear and tear of sensors. A downside of inertial trackers is that they tend to drift due to noise accumulation. Furthermore, errors in tracking systems are present due to different levels of accuracy between sensors. A common approach to overcome these issues, is the fusion of multiple inertial motion devices to increase the accuracy of the tracking and minimise its errors. Several methods have been used for multi-sensor data fusion; however, the simple/extended Kalman filter (SKF/EKF) and their variants have been frequently used for tracking applications. The Kalman filter is an algorithm which operates recursively on streams of noisy data to produce a statistically optimal estimate of the underlying system state [15]. Nevertheless, the inaccuracy of the noise statistical properties largely affects the estimation of the Kalman filter, and even leads to filter divergence [16]. To solve this problem, some researchers have included an adaptive adjustment method based on fuzzy logic in order to improve Kalman filter's estimation accuracy.

In the following sections, we present a MR system that enables remote users to receive assistance when performing onsite activities. The remote assistance platform is divided in two subsystems:

a) An $\mathrm{AR}$ application that allows on-site users to view supplementary information when working with a specific device. The AR application provides sensor data to an adaptive Kalman filter that calculates user's position, based on IMU sensors, to create an accurate representation of user's view.

b) A VR environment that allows experts to supervise the actions taken by users, using a virtual representation of the location, and providing users remote assistance under request. The VR environment allows experts to monitor and control remotely equipment when helping a user.

Moreover, the system implementation includes a full automated temperature control module based on fuzzy logic mechanisms, which is used as part of the overall prototype system to control and monitor the physical object.

\section{THE PROPOSED FUZZY LOGIC BASED SYSTEM FOR MIXED REALITY ASSISTANCE OF REMOTE WORKFORCE}

In previous works [17], [18], we presented a model that allows synchronisation between two or more environments by capturing any changes in physical objects and representing them in a virtual counterpart, achieving bi-directional communication between physical and virtual objects in real time. This is enabled via xReality objects, a type of smart networked object with a virtual representation that is updated and maintained in real-time creating a mirrored state.

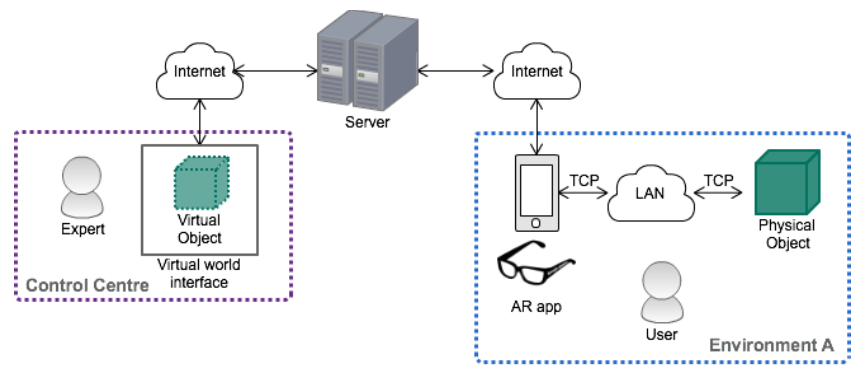

Fig. 2. Mixed Reality Assistance System - Architecture 
Fig. 2 shows the architecture of the proposed MR assistance system. In this architecture, when a worker is performing a scheduled task, he/she is provided with the AR application that can be executed via a mobile phone or a Head Mounted Device (HMD). As shown in Fig.3, this AR application shows additional information related to the physical device he/she is working on, by collecting information of the device's sensors; enabling to monitor the current state of the device (as shown in Fig. 3b). If the user has a problem and needs assistance, the AR application connects directly with the control centre, where an expert user is available to assist with the problem. At that moment, all physical device sensors' data is sent to the control centre, where a $3 \mathrm{D}$ virtual representation of the object is shown in the user interface (as shown in Fig. 3a). This virtual representation is synchronised with the physical object, and shows its current status. Thus, the expert in the control centre has the option to update the status or change the behaviour of any of the actuators in the physical object, in order to help the user to solve the detected problem.

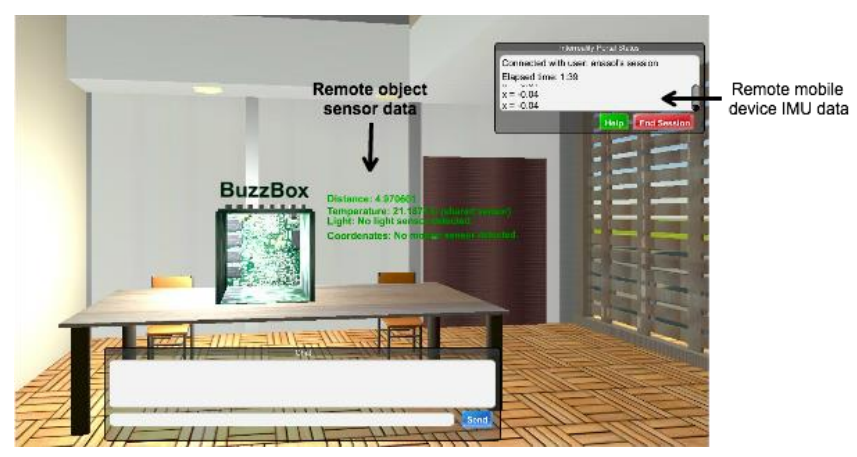

(a)

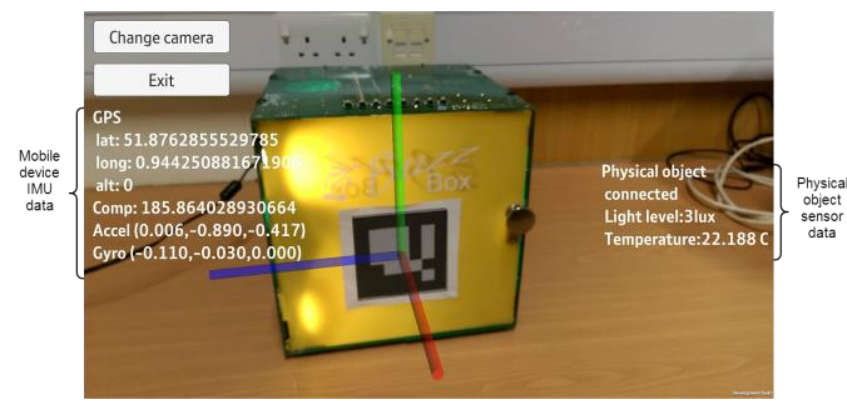

(b)

Fig. 3. Prototype system. a) Virtual representation of physical object and sensor data. b) View from the AR application showing IMU data and object's sensor data (light and temperature sensors).

\section{A. Implementation}

Implementation of the physical device was achieved using Fortito's BuzzBox ${ }^{1}$ (shown in Fig. 3b), a desktop sized emulation of an intelligent environment, designed for education, training and R\&D. The BuzzBox is controlled using a Raspberry $\mathrm{Pi}(\mathrm{RPi})$ via an $\mathrm{I}^{2} \mathrm{C}$ bus, which allows it to connect different embedded sensors and actuators. Raspberry $\mathrm{Pi}^{2}$ is a linux-based credit card sized low-cost computer used for education and embedded systems development worldwide. The BuzzBox contains a variable speed ventilator fan, a controllable heater, 4 dimmable 'warm white' LED lights, 8 push buttons, 8 tricolour LED's, 2 attachable sensors (light and temperature) and an oLED display. For the creation of the virtual representation we used Unity $3 \mathrm{D}^{3}$, a cross-platform game engine for creating interactive 3D content. Finally, synchronisation between environments is achieved using SmartFox Server ${ }^{4}$, a middleware application to create large scale multiplayer games, massively multiplayer online games (MMO) and virtual communities.

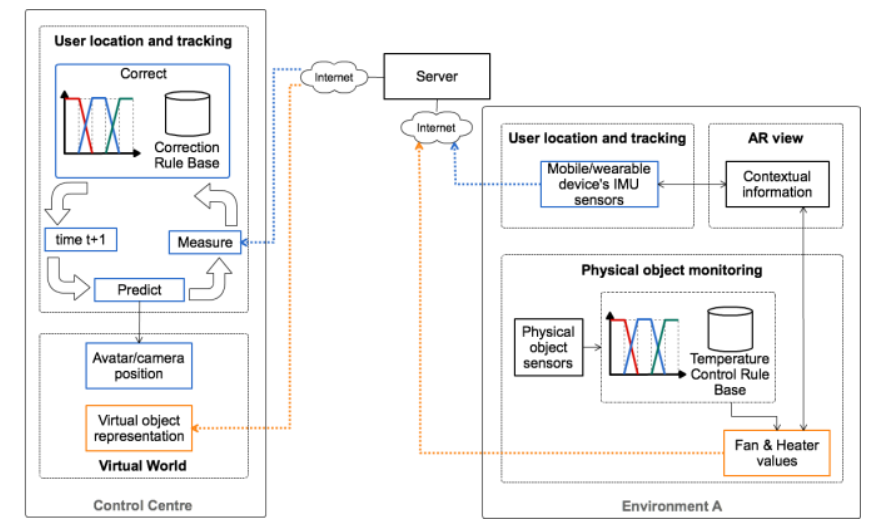

Fig. 4. Mixed Reality Assistance System - Conceptual model

Fig. 4 illustrates the conceptual model of the MR assistance system. Here, system tasks are divided in:

a) Physical object monitoring, which uses a Fuzzy Logic Controller (FLC) to automatically regulate BuzzBox temperature, providing information that is sent to the control centre to be replicated in the $3 \mathrm{D}$ object, synchronising both objects in real time.

b) User location and tracking, which captures mobile/wearable device IMU sensors' data and sends it to the control centre every 0.2 seconds. There, an adaptive Kalman filter calculates user's position and rotation, to then pass these values to the $3 \mathrm{D}$ GUI which moves the camera view within the 3D environment, to replicate user's view. As described in the previous section, knowing the position of the user in an environment is fundamental for allowing the system to display accurately the additional information needed, and to help the expert share the same view as the user.

\section{B. Physical Object Monitoring and Control}

The Fuzzy logic systems (FLS) attempt to mimic human thinking; performing well despite the uncertainty, noise, and imprecision attributed to real-world settings; and playing an important role in modelling and representing imprecise and uncertain linguistic human concepts [19].
1 Fortito Ltd - www.fortito.mx

2 Raspberry Pi Foundation - www.raspberrypi.org
3 Unity 3D - www.unity3d.com

4 SmartFox Server - www.smartfoxserver.com 


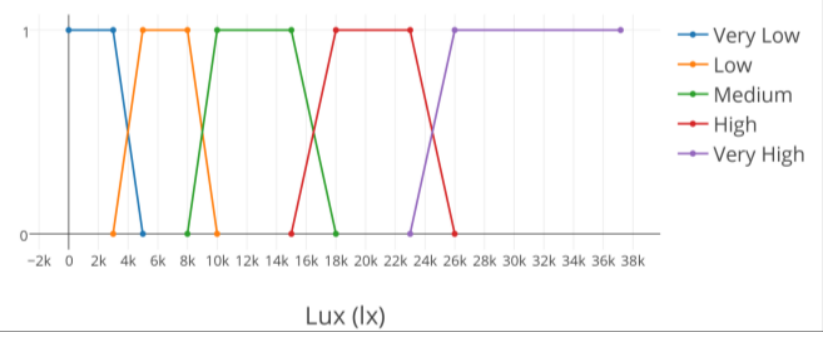

(a)

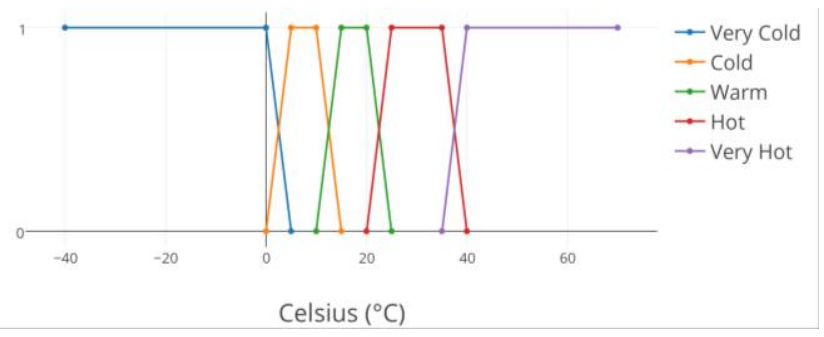

(b)

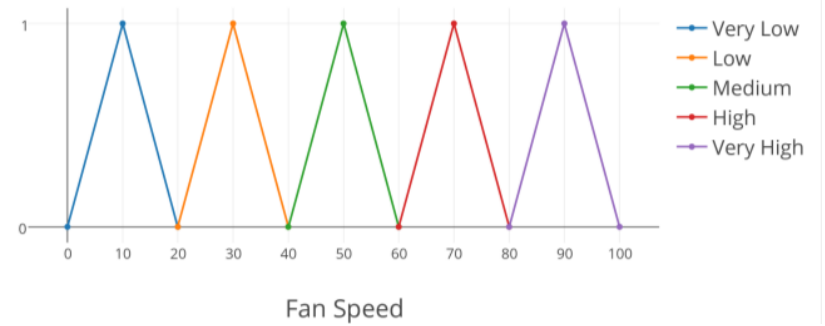

(c)

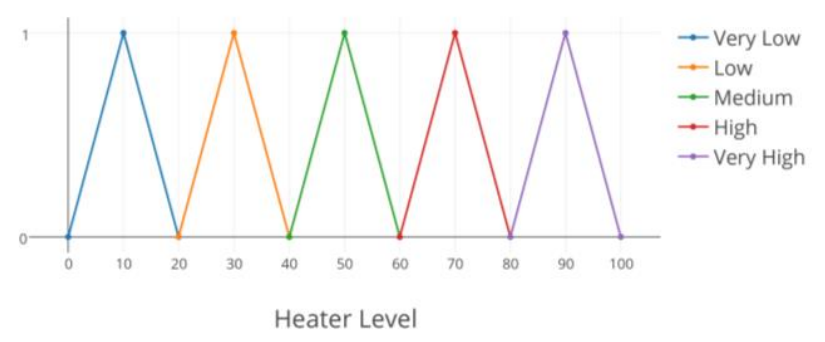

(d)

Fig. 5. Membership functions for BuzzBox's temperature control system. Input: a) Temperature $\left({ }^{\circ} \mathrm{C}\right)$, b) Light Level (lx) Output: c) Fan Speed d) Heater Level

The prototype implementation controls BuzzBox's ventilator and heating system using a type-1 fuzzy logic controller. In this FLC, the fuzzifier maps crisp numerical sensory values into fuzzy sets to activate rules that are determined in linguistic terms [20]. The rule-base (Table I), represented in IF-THEN fuzzy rule format, consists of $5^{2}=25$ rules (five linguistic variables: Very Low, Low, Medium, High, Very High and 2 input variables: temperature and light level).

Each rule has two antecedents with linguistic values from Very High to Very Low for the light sensor (shown in Fig. 5a) and Very Cold to Very Hot for the temperature sensor (shown in Fig. 5b); and, and a consequent that represents a state in the corresponding device (shown in Fig. 5c and Fig. 5d). These rules were designed using a heuristic approach. Therefore, rules (in case of temperature control) can be written as:

IF temperature IS Cold AND light level IS Low

THEN the Fan IS Off

We use the product t-norm to represent the AND logical connective. According to the calculated firing strengths, each input combination is checked with the rule base in order to decide whether a rule will be fired in response to a specific input. When the inference engine returns a result, this result is converted by the defuzzifier into a crisp number; which is taken by the BuzzBox to adjust the level of its temperature control devices (i.e. fan, heater or light level). We use the Height defuzzification in our controllers.

\section{TABLE I. TEMPERATURE CONTROL SOLUTION RULE BASE}

\begin{tabular}{|c|c|c|c|}
\hline \multicolumn{2}{|c|}{ Antecedents } & \multicolumn{2}{|c|}{ Consequents } \\
\hline Temperature & Light level & Fan & Heater \\
\hline Very Cold & Very High & Off & High \\
\hline Very Cold & High & Off & High \\
\hline Very Cold & Medium & Off & High \\
\hline Very Cold & Low & Off & Very High \\
\hline Very Cold & Very Low & Off & Very High \\
\hline Cold & Very High & Off & High \\
\hline Cold & High & Off & High \\
\hline Cold & Medium & Off & High \\
\hline Cold & Low & Off & Very High \\
\hline Cold & Very Low & Off & High \\
\hline Warm & Very High & Medium & Low \\
\hline Warm & High & Medium & Very Low \\
\hline Warm & Medium & Medium & Very Low \\
\hline Warm & Low & Low & Low \\
\hline Warm & Very Low & Low & Low \\
\hline Hot & Very High & Very High & Off \\
\hline Hot & High & Very High & Off \\
\hline Hot & Medium & High & Off \\
\hline Hot & Low & Medium & Off \\
\hline Hot & Very Low & Medium & Off \\
\hline Very Hot & Very High & Very High & Off \\
\hline Very Hot & High & Very High & Off \\
\hline Very Hot & Medium & Very High & Off \\
\hline Very Hot & Low & Very High & Off \\
\hline Very Hot & Very Low & Very High & Off \\
\hline
\end{tabular}


Once a change in any of the BuzzBox devices is detected, the correspondent information is sent to the control centre via the mobile device. Then, the $3 \mathrm{D}$ virtual engine creates a correspondent action to match the physical object current status as shown in Fig, 6a. Because the device updated can only be felt rather than touched or seen, the virtual implementation creates a visual metaphor for the fan (blue sparkles) and heater (red sparkles) as depicted in Fig. $6 \mathrm{~b}$ and $6 \mathrm{c}$ respectively.
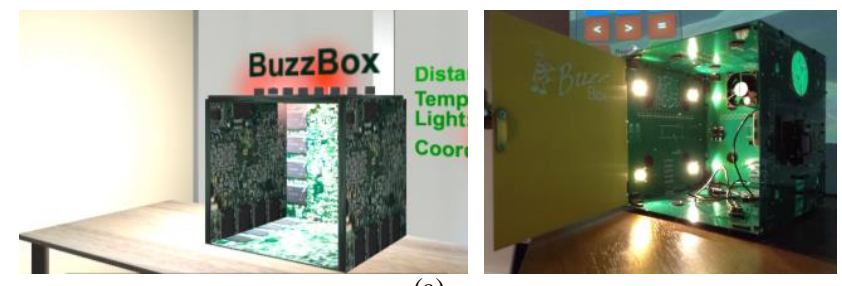

(a)

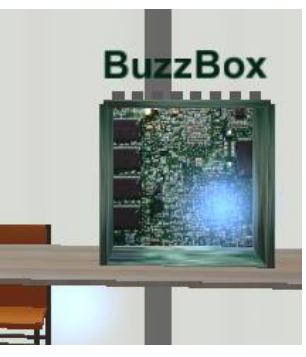

(b)

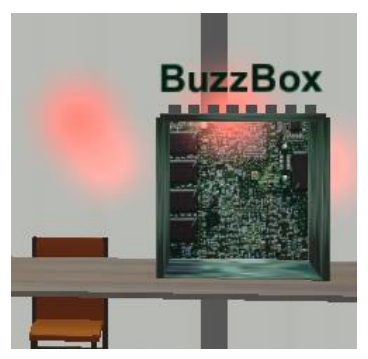

(c)
Fig. 6. a) Prototype system showing synchronised status between the BuzzBox and its virtual representation. b) Fan visual metaphor. c) Heater visual metaphor.

\section{User location and tracking}

To find user's position we used gyroscope and accelerometer data from the mobile device. Once the measurements have been registered by the AR application; sensor data is sent to the $3 \mathrm{D}$ virtual environment implementation, which is the one that will react accordingly to the values received. A well-known problem in the use of IMU sensors is the random noise that they generate. Moreover, the gyro output is influenced by the zero bias and the accelerometer output is affected by gravity acceleration and linear acceleration. These factors influence the accuracy and stability of the measured results.

Due to these facts, we implemented a fuzzy logic based Kalman filter to combine both measurements and improve accuracy. Implementation of the fuzzy logic based Kalman filter was done following the innovation adaptive estimation (IAE) approach (Table II), where the process covariance matrix (Q) and measurement noise covariance matrix (R) adapt themselves as measurements evolve with time [21]. Additionally, we complemented the IAE approach using fuzzy logic techniques following the covariance matching [21] method (as implemented in [22]), where the actual value of the covariance of the innovation sequences is adjusted according to its match to the theoretical value.
TABLE II. ADAPTIVE KALMAN FILTER (IAE)

\begin{tabular}{|c|c|}
\hline $\begin{array}{c}\text { Time Update } \\
\text { (prediction) }\end{array}$ & $\begin{array}{c}\text { Measurement Update } \\
\text { (correction) }\end{array}$ \\
\hline$\hat{x}^{-}{ }_{k+1}=A_{k} \hat{x}_{k}+B_{k} u_{k}$ & $K_{k}=P^{-}{ }_{k} H_{k}{ }^{T}\left[H_{k} P^{-}{ }_{k} H_{k}{ }^{T}+R_{k}\right]^{-1}$ \\
\hline \multirow{2}{*}{$P_{k+1}^{-}=A_{k} P_{k} A_{k}^{T}+Q_{k}$} & $\hat{x}_{k}=\hat{x}_{k}^{-}+K_{k}\left[z_{k}-H_{k} \hat{x}_{k}^{-}\right]$ \\
\hline & $P_{k}=\left[I-K_{k} H_{k}\right] P_{k}^{-}$ \\
\hline
\end{tabular}

Table II shows the structure of the adaptive Kalman filter. Where $x_{k}$ is a $n \times 1$ system state vector, $A_{k}$ is a $n x n$ transition matrix, $u_{k}$ is a $l x 1$ vector of the input forcing function, $B_{k}$ is an $n x l$ matrix, $w_{k}$ is a $n x 1$ process noise vector, $z_{k}$ is a $m \times 1$ measurement vector, $H_{k}$ is a $m x n$ measurement matrix and $v_{k}$ is a $m x 1$ measurement noise vector.

The actual covariance is defined as an approximation of the Inn $_{k}$ sample covariance through averaging inside a moving estimation window of size M:

$$
\hat{C}_{\text {Inn }_{k}}=\frac{1}{M} \sum_{j=j_{0}}^{k} \operatorname{Inn}_{k} \operatorname{Inn} n_{k}^{T}
$$

Where $j_{0}=k-M+1$ is the first sample inside the estimation window.

The theoretical covariance of the innovation sequence is defined as:

$$
S_{k}=H_{k} P_{k}^{-} H_{k}^{T}+R_{k}
$$

Thus, if the actual covariance value $\hat{C}_{I n n_{k}}$ (2) is within the range predicted by the theoretical covariance of the innovation sequence $S_{k}$ (3) and the difference is very near to zero, then both covariances' values are close to each other, no change on the value $\mathrm{R}$ is needed. If the actual covariance $\hat{C}_{I n n_{k}}(2)$ is greater than its theoretical value $S_{k}$ (3), the value of $\mathrm{R}$ should be decreased. On the contrary, if $\hat{C}_{I n n_{k}}$ (2) is less than $S_{k}$ (3); the value of $\mathrm{R}$ should be increased. This is described with the FLC rule base in table III.

An extra factor to be considered is that the proposed architecture adds an additional impression level, due to the fact that IMU sensor data is captured in the mobile/wearable device but processed by the adaptive Kalman filter only after being received in the $3 \mathrm{D}$ virtual control centre application. This approach was taken to reduce the computation load on the mobile device and provide a better user experience in the AR application, avoiding bottlenecks and sluggish responses. However, this also includes an additional factor of uncertainty due to diverse network issues that can be present, such as network latency and packet loss, which could affect the result of the prediction generated by the adaptive Kalman filter. Thus, a calculation between the time elapsed between the current received data and the previous received data is considered in the FLC rule base (4).

$$
n_{\Delta t}=n_{t}-n_{t-1}
$$


If the time difference is high then there was a possible problem in the sending/reception of information through the network and some data was lost. If the time difference is close to zero it means that the value has not been updated yet. And finally, if the time difference is low it means that we are working with a new value sent by the AR application.

TABLE III. ADAPTIVE KALMAN FILTER RULE BASE

\begin{tabular}{|c|l|c|}
\hline \multicolumn{2}{|c|}{ Antecedents } & Consequents \\
\hline $\mathbf{n}_{\Delta \mathrm{t}}$ & $\widehat{\boldsymbol{C}}_{\text {In }_{\boldsymbol{k}}}-\boldsymbol{S}_{\boldsymbol{k}}$ & $\boldsymbol{R}_{\boldsymbol{k}}$ \\
\hline HIGH & POSITIVE & MAINTAIN \\
\hline HIGH & ZERO & MAINTAIN \\
\hline HIGH & NEGATIVE & MAINTAIN \\
\hline ZERO & POSITIVE & MAINTAIN \\
\hline ZERO & ZERO & MAINTAIN \\
\hline ZERO & NEGATIVE & MAINTAIN \\
\hline LOW & POSITIVE & DECREASE \\
\hline LOW & ZERO & MAINTAIN \\
\hline LOW & NEGATIVE & INCREASE \\
\hline
\end{tabular}

Thus $\mathrm{R}$ is adjusted according to:

$$
R_{k}=R_{k-1}+\Delta R_{k}
$$

Where $\Delta R_{k}$ is added or subtracted from $\mathrm{R}$ at each instant of time. The membership functions for the fuzzy sets representing the inputs and outputs of the FLC based Kalman filter are shown in Fig. 7.

\section{EXPERIMENTS AND RESULTS}

Our prototype system shown in Fig. 8 was deployed on an immersive environment using Immersive Display's ImmersaVu $\mathrm{u}^{5}$ screen (shown in Fig. 8b) which is a composite moulded panoramic dome screen $\left(75^{\circ} \times 180^{\circ}\right.$ wide field of view $($ FOV $)$ ), which allows a free range of head movement, along with providing an increased feeling of immersion when in comparison to traditional screens. Immersion can be defined as "a set of physical properties that give rise to presence" [23]. Presence, is the sense of being in a place or environment [24], and it could be considered as the key concept that allows defining virtual reality in terms of human experience rather than technological hardware. However, it is directly dependant on the perceptual feedback the user receives via the appropriate technology [23]. Thus, we used this setting to provide the expert in the control centre with an enhanced view of the object according to user's position.
The user was provided with a mobile device HTC One m8, with a Quad-core $2.3 \mathrm{GHz}$ processor, 16/32 GB of internal memory, 2 GB RAM, accelerometer, gyroscope, and Android OS, v4.4.2 (KitKat).

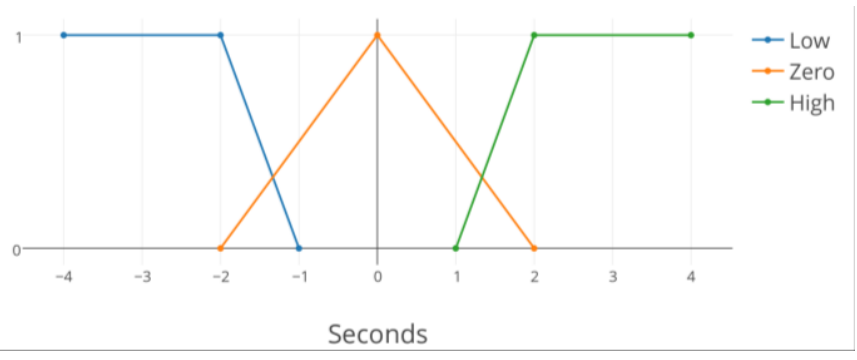

(a)

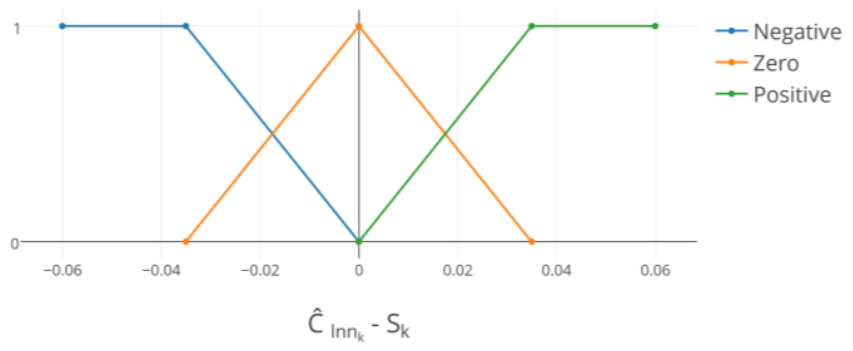

(b)

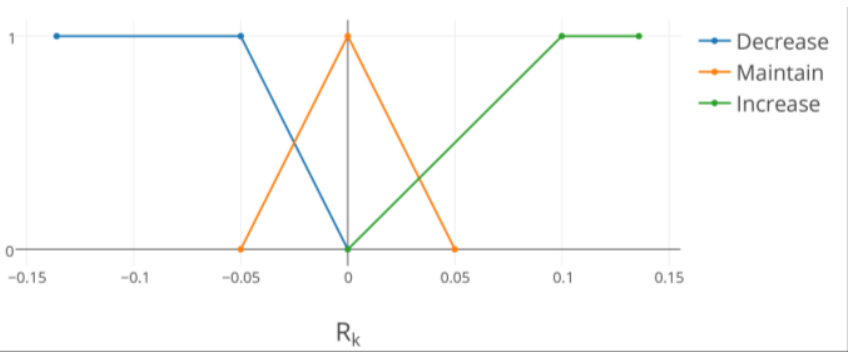

(c)

Fig. 7. Membership functions for adaptive Kalman filter implementation. Input: a) Network delay, b) Actual covariance minus theoretical covariance $\left(\widehat{\boldsymbol{C}}_{\boldsymbol{I n n} \boldsymbol{n}_{\boldsymbol{k}}}-\mathbf{S}_{\boldsymbol{k}}\right)$. Output: c) Measurement noise covariance matrix $\left(\mathrm{R}_{\mathrm{k}}\right)$ 


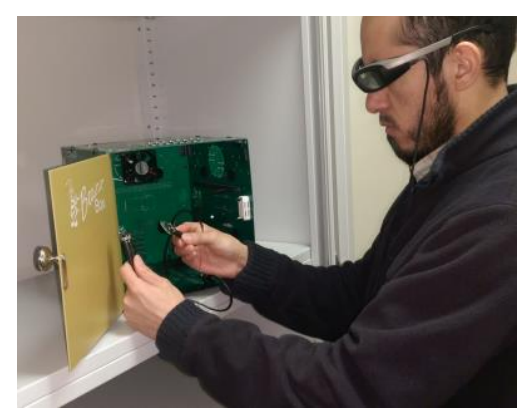

(a)

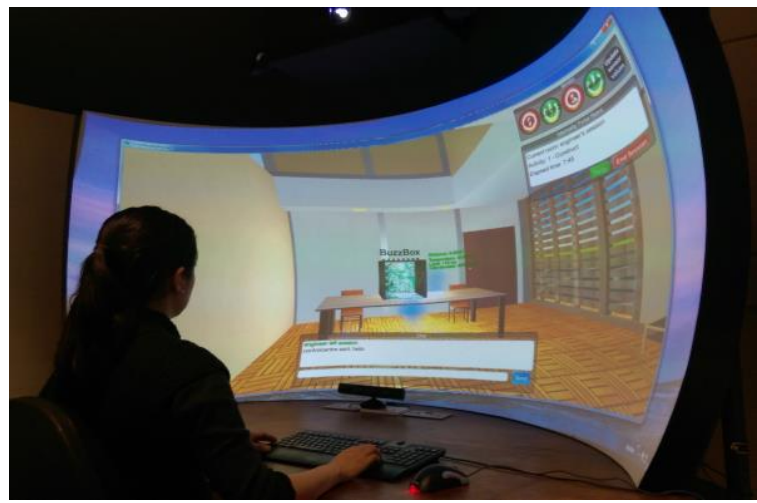

(b)

Fig. 8. Experimental scenario. a) Technician b) Control Centre

Experiments were carried indoors using an area of 450x450 $\mathrm{cm}^{2}$, defining a pair of coordinates $(\mathrm{x}, \mathrm{y})$ every 50 centimetres. This was done to define benchmark coordinates and to create a predefined scale with the simulated environment. Fig. 9 shows the results comparison between the predefined benchmark coordinates and: a) result coordinates from the adaptive fuzzy based Kalman filter, b) values obtained from the IMU devices.

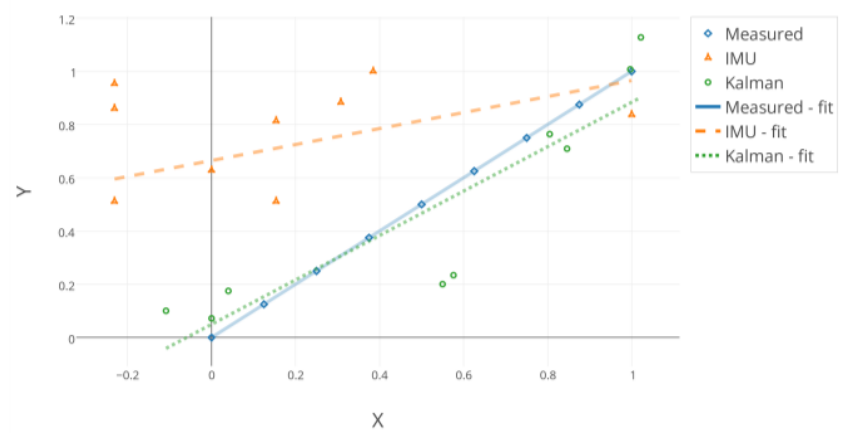

Fig. 9. Comparison between real world benchmark coordinates, Kalman filter results and IMU data.
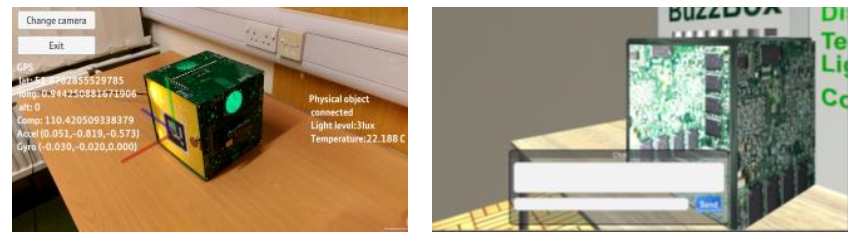

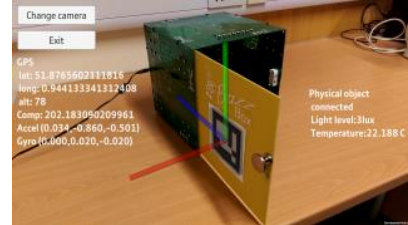

(a)

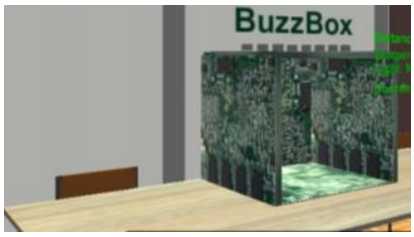

(b)
Fig. 10. Views from the a) AR application and b) VR Control Centre application

It is clear that the results from the fuzzy logic based Kalman filter has shown a promising result in improving the estimation and enhancing the level of accuracy with respect to the real world coordinates, compared to the data obtained from the sensors. These results show an accuracy improvement (measured via RMSE) of $70.13 \%$ compared to IMU sensor data.

TABLE IV. RESULTS

\begin{tabular}{|l|c|c|}
\hline \multicolumn{1}{|c|}{ Comparison } & MSE & $\boldsymbol{R M S E}$ \\
\hline Measured vs. IMU data & 0.27688 & 0.52619 \\
\hline Measured vs. Kalman filter data & 0.02471 & 0.15720 \\
\hline
\end{tabular}

Fig. 10 shows various experiments which shows how the AR systems in the control centre reflects the actions of the real world situation and enables to help the field workforce.

\section{CONCluSiONS AND FUTURE WORK}

In this paper, we have proposed an innovative approach that combines a mixed reality system with fuzzy logic mechanisms to create a platform to assist remote workers in their daily activities. The infrastructure described here is based on two elements:

a) An augmented reality client application, which provides users with access to physical devices' information, enabling additional contextual information; and opening communication between the remote user and a control centre, where expert users would be able to assist the remote user when required.

b) A virtual reality control centre, able to reproduce in real time, sensor information sent by the augmented reality client application, using virtual objects. Additionally, the control centre application enables the monitoring and control of the remote physical object, allowing the expert to have a better understanding of the situation experienced by the user. 
Furthermore, the proposed system is supported by fuzzy logic mechanisms that improve accuracy in user tracking and object monitoring, fundamental for the correct blending between virtual and physical elements present in a mixed reality environment. In particular, the adaptive fuzzy logic based Kalman filter showed an accuracy improvement of $70.13 \%$ compared to IMU sensor data.

As part of the future work, we will define specific scenarios to implement diverse automated assistance tasks, where the user could be guided by the AR application to achieve simple tasks; asking for expert's guidance only after the indications of the automated system had been proved no useful to solve a particular situation. Similarly, we will continue developing visual tools for the expert to point specific areas or elements in the monitored device(s) where the user needs to focus to solve a particular issue. Additionally, we will explore the use of the proposed system with different head mounted devices, to enhance users' feeling of immersion, and provide interaction with the overall platform via a hands-free interface.

\section{REFERENCES}

[1] M. Hincapié, A. Caponio, H. Rios and E. González Mendívil, "An introduction to Augmented Reality with applications in aeronautical maintenance," in 13th International Conference on Transparent Optical Networks (ICTON), Stockholm, 2011.

[2] Y.-S. Gloy, D. Kerpen, J. Lemm, A. Hansen-Ampah, M. Saggiomo and M. Lohrer, "SozioTex-Sociotechnical systems in the Textile Industry: Interdisciplinary Competence Build-up in Human-machine Interaction Facing Demographic Change," Textile Science \& Engineering, vol. 5, no. 6, 2015.

[3] M. Rosenthal, A. State, J. Lee, G. Hirota, J. Ackerman, K. Keller, E. Pisano, M. Jiroutek, K. Muller and H. Fuchs, "Augmented reality guidance for needle biopsies: an initial randomized, controlled trial in phantoms," in Medical Image Computing and Computer-Assisted Intervention (MICCAI) , Utrecht, 2001.

[4] S. K. Ong and A. Y. C. Nee, Virtual and augmented reality applications in manufacturing, Springer Science \& Business Media, 2013.

[5] A. Sarwal, C. Baker and D. Filipovic, "Head-worn display-based augmented reality system for manufacturing," Defense and Security. International Society for Optics and Photonics., pp. 115-122, 2005.

[6] M. Livingston, L. Rosenblum, S. Julier, D. Brown, Y. Baillot, J. E. Swan II, J. Gabbard, Y. Baillot and D. Hix, "An augmented reality system for military operations in urban terrain," in Interservice / Industry Training, Simulation, \& Education Conference (I/ITSEC '02), Orlando, 2002.

[7] A. Webster, S. Feiner, B. MacIntyre, W. Massie and T. Krueger, "Augmented reality in architectural construction, inspection and renovation," in ASCE Third Congress on Computing in Civil Engineering, 1996.
[8] P. Milgram and F. Kishino, "A taxonomy of virtual reality displays," IEICE TRANSACTIONS on Information and Systems, Vols. E77-D, no. 12, pp. 1321-1329, 1994.

[9] S. Pastoor and C. Conomis, "Mixed Reality Displays," in $3 D$ Videocommunication: Algorithms, Concepts and Real-Time Systems in Human Centred Communication, John Wiley \& Sons, 2005, pp. 261-280.

[10] S. Ellis, "What are virtual environments?," IEEE Computer Graphics and Applications, vol. 14, no. 1, 1994.

[11] R. Azuma, "A survey of augmented reality," Presence: Teleoperators and Virtual Environments, vol. 6, no. 4, p. 355-385, 1997.

[12] F. Zhou, H. Been-Lirn Duh and M. Billinghurst, "Trends in augmented reality tracking, interaction and display: A review of ten years of ISMAR," in Proceedings of the 7th IEEE/ACM International Symposium on Mixed and Augmented Reality, Washington, 2008.

[13] E. Bostanci, N. Kanwal, S. Ehsan and A. F. Clark, "User tracking methods for augmented reality," International Journal of Computer Theory and Engineering, vol. 5, no. 1, p. 93-98, 2013.

[14] J. P. Rolland, L. Davis and Y. Baillot, "A survey of tracking technology for virtual environments," in Fundamentals of wearable computers and augmented reality, vol. 1, 2001, pp. 67-112.

[15] R. E. Kalman, "A new approach to linear filtering and prediction problems," Journal of Fluids Engineering, vol. 82, no. 1, pp. 35-45, 1960.

[16] M. Jin, J. Zhao, J. Jin, G. Yu and W. Li, "The adaptive Kalman filter based on fuzzy logic for inertial motion capture system," Measurement, no. 49 , pp. 196-204, 2014.

[17] A. Peña-Rios, V. Callaghan, M. Gardner and M. Alhaddad, "Using mixed-reality to develop smart environments," in 10th International Conference on Intelligent Environments 2014 (IE'14), Shanghai, 2014.

[18] A. Peña-Rios, V. Callaghan, M. Gardner and M. Alhaddad, "Developing xReality objects for mixed-reality environments," in Workshop Proceedings of the 9th International Conference on Intelligent Environments, Athens, 2013.

[19] H. Hagras, "Embedding Computational Intelligence in Pervasive Spaces," IEEE Pervasive Computing, vol. 6, no. 3, pp. 85-89, 2007.

[20] J. Mendel, Uncertain Rule-Based Fuzzy Logic Systems: Introduction and New Directions, Upper Saddle River, NJ: Prentice Hall Inc., 2001.

[21] R. K. Mehra, "On-line identification of linear dynamic systems with applications to Kalman filtering," IEEE Transactions on Automatic Control, Vols. AC-16, no. 1, pp. 12-21, 1971.

[22] D. Loebis, R. Sutton, J. Chudley and W. Naeem, "Adaptive tuning of a Kalman filter via fuzzy logic for an intelligent," Control Engineering Practice, vol. 12, p. 1531-1539, 2004.

[23] W. A. Ijsselsteijn, "History of Telepresence," in $3 D$ Videocommunication: Algorithms, Concepts and Real-Time Systems in Human Centred Communication, 2006, pp. 5-21.

[24] M. Slater and S. Wilbur, "A framework for immersive virtual environments (FIVE): Speculations on the role of presence in virtual environments," Presence: Teleoperators and virtual environments, vol. 6, no. 6, pp. 603-616, 1997. 